\title{
Oligodendrogliomas, IDH-mutant and 1p/ 19q-codeleted, arising during teenage years often lack TERT promoter mutation that is typical of their adult counterparts
}

\author{
Julieann Lee ${ }^{1}$, Angelica R. Putnam², Samuel H. Chesier ${ }^{3}$, Anuradha Banerjee ${ }^{4}$, Corey Raffel ${ }^{5}$, Jessica Van Ziffle ${ }^{1,6}$, \\ Courtney Onodera ${ }^{1,6}$, James P. Grenert ${ }^{1,6}$, Boris C. Bastian ${ }^{1,6}$, Arie Perry ${ }^{1,5}$ and David A. Solomon ${ }^{1,6^{*}}$ (D)
}

Keywords: Oligodendroglioma, IDH mutation, 1p/19q-codeletion, Teenager, Pediatric, TERT promoter, FGFR1, CIC, IDH1, Molecular neuro-oncology

The genetic alterations that characterize oligodendroglial neoplasms have been defined over the past decade. In adults, oligodendrogliomas are genetically defined by the combination of IDH1 p.R132 or IDH2 p.R172 mutation, TERT promoter hotspot mutation (either c.-124C $>\mathrm{T}$ or c. $-126 \mathrm{C}>\mathrm{T}$ ), and chromosomes $1 \mathrm{p}$ and $19 \mathrm{q}$ co-deletion, which is frequently accompanied by mutations involving CIC, FUBP1, TCF12, NOTCH1, and PIK3CA genes [2, 3, 7, $13,21]$. Oligodendrogliomas in children often lack the IDH mutation, TERT promoter mutation, and 1p/19q-codeletion that is observed in their adult counterparts [14, 20]. Instead, they most commonly harbor solitary pathogenic alterations in the FGFR1 oncogene that cause constitutive activation of the kinase domain via gene fusion, tandem duplication, or missense mutations that localize at one of two hotspots (p.N546 or p.K656) $[18,23]$.

To investigate the molecular pathogenesis of oligodendrogliomas arising during teenage years, we assembled a cohort of tissue specimens from three patients (Fig. 1a). The one male and two female patients ranged in age at time of initial surgery from 10-18 years. All patients presented with headaches that led to brain imaging, which demonstrated non-enhancing, T2-hyperintense masses centered in the frontal $(n=2)$ or parietal $(n=1)$ lobes (Fig. 1b). All cases were histologically characterized by an infiltrative glial neoplasm composed of cells with uniform round

\footnotetext{
* Correspondence: david.solomon@ucsf.edu

${ }^{1}$ Department of Pathology, University of California, San Francisco, CA, USA ${ }^{6}$ Clinical Cancer Genomics Laboratory, University of California, San Francisco, CA, USA

Full list of author information is available at the end of the article
}

nuclei containing delicate chromatin (Fig. 1c). Mitoses were inconspicuous, and neither microvascular proliferation nor necrosis were present. Immunohistochemistry revealed that the tumor cells were OLIG2 positive, had intact/retained nuclear expression of ATRX protein, and showed only occasional positivity for $\mathrm{p} 53$ protein. The Ki-67 labeling index was uniformly low (less than $2 \%$ ).

Genomic DNA was extracted from formalin-fixed, paraffin-embedded tumor tissue, and targeted capturebased next-generation DNA sequencing was performed as previously described using the UCSF500 Cancer Panel $[8,9,11,16,17]$, which assesses approximately 500 cancer-associated genes for mutations, copy number alterations, and structural variants including gene fusions (Additional file 1: Table S1). All three cases demonstrated IDH mutation, with two harboring IDH2 p.R172K and one harboring IDH1 p.R132H (Additional file 1: Table $\mathrm{S} 2$ and Additional file 2: Fig. S1). Additionally, case \#1 contained a damaging missense mutation in TP53 (p.R175H), which was present at a subclonal allele frequency $(6 \%)$ relative to the IDH1 mutation (20\%), indicating that it was only present in a subset of tumor cells. Case \#3 additionally contained a truncating nonsense mutation in the CIC tumor suppressor gene (p.S349*). No pathogenic mutations were identified involving any of the other genes targeted for sequencing by this assay. Chromosomal copy number analysis revealed losses of $1 \mathrm{p}$ and $19 \mathrm{q}$ in all three cases, which uniformly involved the entire arms of these chromosomes. No other chromosomal gains, losses, or focal amplifications or 


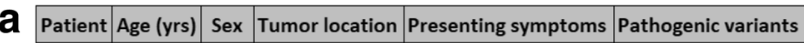

\begin{tabular}{|c|c|c|l|l|l|}
\hline$\# 1$ & 10 & $\mathrm{~F}$ & right frontal & headaches, nausea & IDH1 p.R132H, 1p/19q-codeletion, TP53 p.R175H (subclonal) \\
\hline$\# 2$ & 17 & M & left parietal & headaches, nausea & IDH2 p.R172K, 1p/19q-codeletion \\
\hline$\# 3$ & 18 & $\mathrm{~F}$ & left frontal & headaches & IDH2 p.R172K, 1p/19q-codeletion, CIC p.S349* \\
\hline
\end{tabular}

b 弗
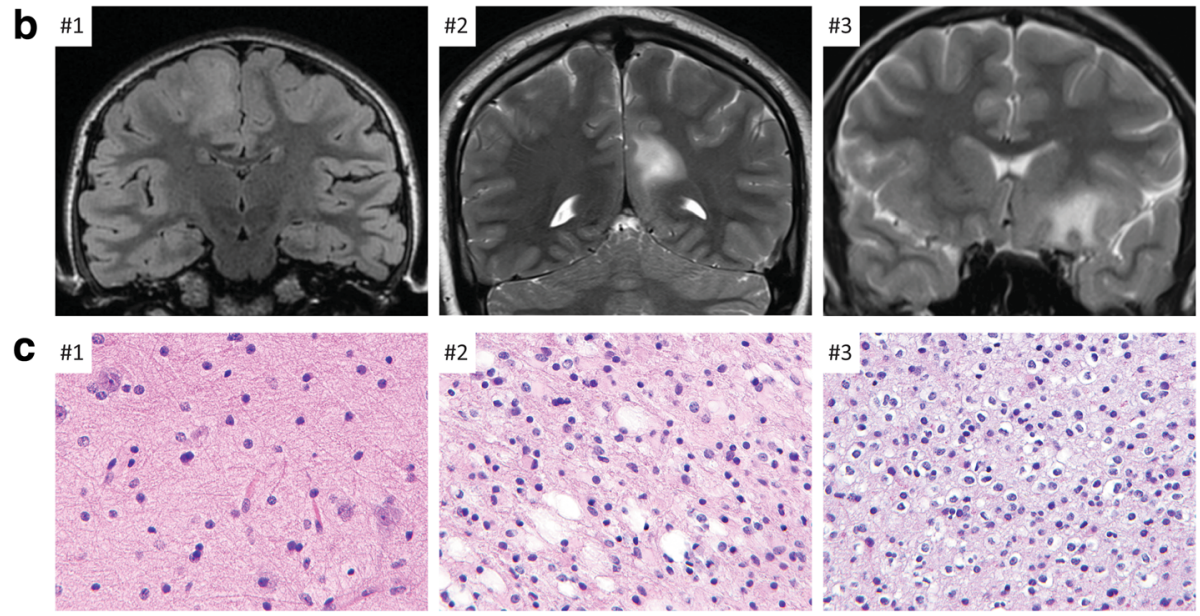

d

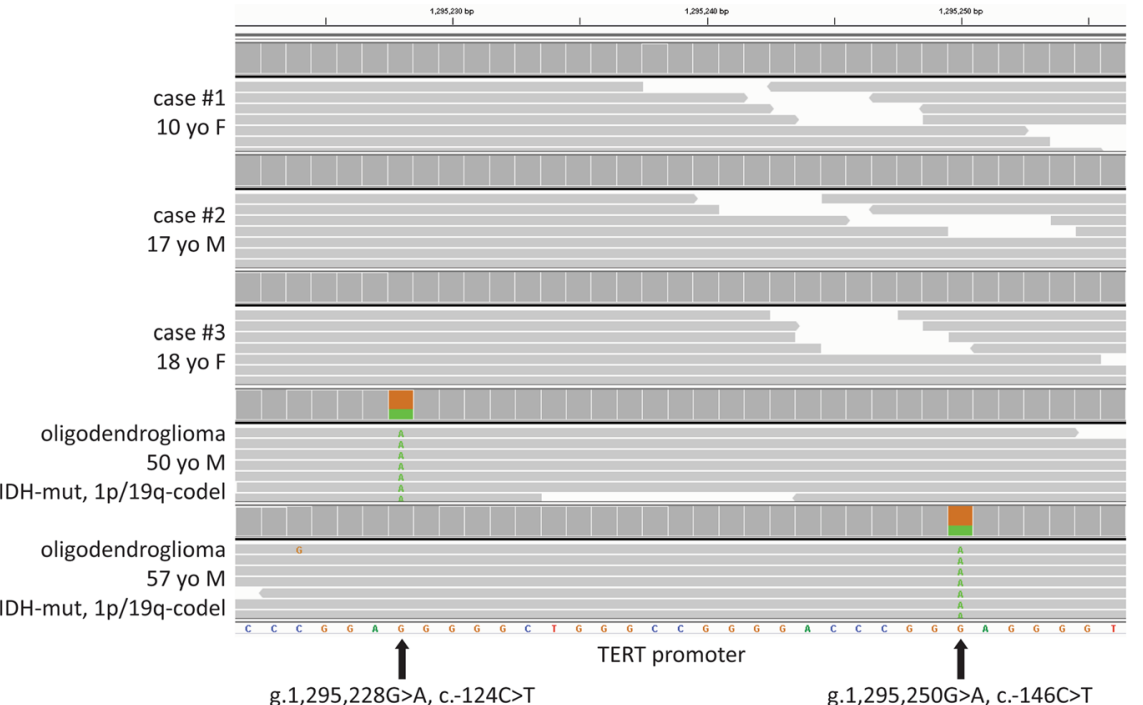

Fig. 1 Oligodendrogliomas, IDH-mutant and 1p/19q-codeleted, arising during teenage years often lack TERT promoter hotspot mutation. a, Table of the clinicopathologic features of the three teenagers with oligodendroglioma. $\mathbf{b}$, Pre-operative magnetic resonance imaging for the three patients. $\mathbf{c}$, Histology of the three tumors. Hematoxylin and eosin staining, 60x magnification. $\mathbf{d}$, Snapshot from the Integrated Genome Viewer for the three oligodendrogliomas in teenagers demonstrating absence of the TERT promoter hotspot mutation seen in oligodendrogliomas, IDH-mutant and 1p/ 19q-codeleted, from adults

deletions were identified in any of the tumors (Additional file 1: Table S3 and Additional file 2: Fig. S2). Notably, all three cases lacked mutation at either of the two hotspots in the promoter region of the TERT gene (Fig. 1d), and also did not harbor either TERT gene amplification or structural rearrangement within the $50 \mathrm{~Kb}$ of upstream sequence covered by this assay, where rearrangements are commonly found in high-risk neuroblastomas, chromophobe renal cell carcinomas, and IDH-wildtype glioblastomas lacking TERT promoter hotspot mutation $[5,6,15,22]$.
In order to further assess the frequency of TERT promoter mutation in IDH-mutant and 1p/19q-codeleted oligodendrogliomas in teenagers, we examined data from the four major pediatric low-grade glioma genomics studies published to date $[1,18,19,23]$. Together, these four studies included only a single teenage patient with an oligodendroglioma, IDH-mutant and $1 \mathrm{p} / 19 \mathrm{q}$-codeleted, in which TERT promoter status had been assessed. This patient (SJLGG034 from the Zhang et al study, also labeled LGNT20 in the Qaddoumi et al study) was a 15 year old male with an oligodendroglioma that harbored 
IDH1 p.R132H mutation, multiple CIC mutations, $1 \mathrm{p} /$ $19 \mathrm{q}$-codeletion, and reportedly lacked TERT promoter mutation $[18,23]$. We next examined data from the most recent glioma metagenomics study by The Cancer Genome Atlas Research Network that included 89 cases of oligodendroglioma, IDH-mutant and $1 \mathrm{p} / 19 \mathrm{q}$-codeleted, WHO grade II or III, in which TERT promoter status was reported [4]. 87 of these 89 cases (98\%) reportedly harbored TERT promoter hotspot mutation and were all in adults (age range 20-75 years at diagnosis). Two of the 89 cases are reported to be TERT promoter wildtype, one in a teenager and one in an older adult. The first TERT promoter wildtype oligodendroglioma case (TCGA-DB-5278) was centered in the left frontal lobe of a 17 year old male who had presented with seizures, demonstrated WHO grade II histologic features, IDH1 p.R132H mutation, CIC mutation, $1 \mathrm{p} / 19 \mathrm{q}$-codeletion, and did not have TERT overexpression. The second TERT promoter wildtype oligodendroglioma case (TCGA-HT-8010) was in a 64 year old female, had WHO grade II histologic features, IDH mutation, NF1 mutation, $1 \mathrm{p} / 19 \mathrm{q}$-codeletion, and also did not have TERT overexpression. Thus, according to the latest published dataset from The Cancer Genome Atlas, TERT promoter mutation was present in $87 / 88$ cases $(99 \%)$ of IDH-mutant and $1 \mathrm{p} / 19 \mathrm{q}$-codeleted oligodendrogliomas in adults age $20+$ years at time of diagnosis. In contrast, TERT promoter mutation was present in $0 / 5$ cases $(0 \%)$ of IDH-mutant and 1p/19-codeleted oligodendrogliomas in teenagers, including the three patients from our cohort, one patient from Zhang et al, and one patient from The Cancer Genome Atlas.

Together, these findings suggest that oligodendrogliomas arising during teenage years are genetically distinct from their adult counterparts based on the absence of TERT promoter mutation. Though telomere maintenance has been proposed as a requirement for gliomagenesis in adults $[10,12]$, it does not appear to be necessary in oligodendrogliomas, IDH-mutant and 1p/19q-codeleted, in teenagers. We speculate that this may be due to the low number of cell divisions that have taken place in oligodendrogliomas arising in teenagers relative to adults such that selection pressure for acquisition of telomere maintenance mechanism has not yet occurred. The absence of telomerase activation in these tumors may potentially correlate with the less frequent anaplasia and more indolent clinical behavior that has been observed in pediatric oligodendrogliomas compared to their adult counterparts [20].

\section{Additional files}

Additional file 1: Table S1. List of the 479 genes targeted for sequencing on the UCSF500 Cancer Panel. Table S2. Pathogenic mutations identified in the three oligodendrogliomas. (XLSX $20 \mathrm{~kb})$
Additional file 2: Figure S1. Snapshots from the Integrated Genome Viewer of the IDH mutations present in each of the three oligodendrogliomas, IDH-mutant and 1p/19q-codeleted, in teenagers. Fig. S2. Chromosomal copy number and zygosity plots for the oligodendroglioma, IDHmutant and 1p/ 19q-codeleted, from teenage patient \#2. (PDF $950 \mathrm{~kb}$ )

\section{Acknowledgements}

B.C.B. is supported by an NCl Outstanding Investigator Award (R35 CA220481). D.A.S. is supported by NIH Director's Early Independence Award (DP5 OD021403) and the UCSF Physician-Scientist Scholar Program.

\section{Availability of data and materials}

Sequencing data files are available from the authors upon request.

\section{Authors' contributions}

$J$, ARP, AP, and DAS performed pathologic assessment. SHC and CR provided neurosugical management. AB provided neuro-oncology management. JVZ, CO, JPG, BB, and DAS performed genomic analysis. JL and DAS wrote the manuscript and created the figures. All authors read and approved the final manuscript.

\section{Ethics approval and consent to participate}

This study was approved by the Committee on Human Research of the University of California, San Francisco, with a waiver of patient consent.

Consent for publication

Not applicable.

Competing interests

The authors declare that they have no competing interests related to this study.

\section{Publisher's Note}

Springer Nature remains neutral with regard to jurisdictional claims in published maps and institutional affiliations.

\section{Author details}

'Department of Pathology, University of California, San Francisco, CA, USA. ${ }^{2}$ Department of Pathology, University of Utah School of Medicine, Salt Lake City, UT, USA. ${ }^{3}$ Department of Neurosurgery, University of Utah School of Medicine, Salt Lake City, UT, USA. ${ }^{4}$ Division of Hematology/Oncology, Department of Pediatrics, University of California, San Francisco, CA, USA. ${ }^{5}$ Department of Neurological Surgery, University of California, San Francisco, CA, USA. ${ }^{6}$ Clinical Cancer Genomics Laboratory, University of California, San Francisco, CA, USA.

Received: 11 September 2018 Accepted: 11 September 2018

Published online: 19 September 2018

\section{References}

1. Bandopadhayay P, Ramkissoon LA, Jain P et al (2016) MYB-QKI rearrangements in angiocentric glioma drive tumorigenicity through a tripartite mechanism. Nat Genet 48:273-282

2. Bettegowda C, Agrawal N, Jiao Y et al (2011) Mutations in CIC and FUBP1 contribute to human oligodendroglioma. Science 333:1453-1455

3. Cancer Genome Atlas Research Network (2015) Comprehensive, integrative genomic analysis of diffuse lower-grade gliomas. N Engl J Med 372:24812498

4. Ceccarelli M, Barthel FP, Malta TM et al (2016) Molecular profiling reveals biologically discrete subsets and pathways of progression in diffuse glioma. Cell 164:550-563

5. Davis CF, Ricketts CJ, Wang M et al (2014) The somatic genomic landscape of chromophobe renal cell carcinoma. Cancer Cell 26:319-330

6. Diplas BH, He X, Brosnan-Cashman JA et al (2018) The genomic landscape of TERT promoter wildtype-IDH wildtype glioblastoma. Nat Commun 9:2087

7. Eckel-Passow JE, Lachance DH, Molinaro AM et al (2015) Glioma groups based on 1p/19q, IDH, and TERT promoter mutations in tumors. N Engl J Med 372:2499-2508 
8. Goode B, Mondal G, Hyun M et al (2018) A recurrent kinase domain mutation in PRKCA defines chordoid glioma of the third ventricle. Nat Commun 9:810

9. Iorgulescu JB, Van Ziffle J, Stevers M et al (2018) Deep sequencing of WNTactivated medulloblastomas reveals secondary SHH pathway activation. Acta Neuropathol 135:635-638

10. Killela PJ, Reitman ZJ, Jiao Y et al (2013) TERT promoter mutations occur frequently in gliomas and a subset of tumors derived from cells with low rates of self-renewal. Proc Natl Acad Sci U S A 110:6021-6026

11. Kline CN, Joseph NM, Grenert JP et al (2017) Targeted next-generation sequencing of pediatric neuro-oncology patients improves diagnosis, identifies pathogenic germline mutations, and directs targeted therapy. Neuro-Oncol 19:699-709

12. Koelsche C, Sahm F, Capper D et al (2013) Distribution of TERT promoter mutations in pediatric and adult tumors of the nervous system. Acta Neuropathol 126:907-915

13. Labreche K, Simeonova I, Kamoun A et al (2015) TCF12 is mutated in anaplastic oligodendroglioma. Nat Commun 6:7207

14. Nauen D, Haley L, Lin MT et al (2016) Molecular analysis of pediatric oligodendrogliomas highlights genetic differences with adult counterparts and other pediatric gliomas. Brain Pathol 26:206-214

15. Peifer M, Hertwig F, Roels F et al (2015) Telomerase activation by genomic rearrangements in high-risk neuroblastoma. Nature 526:700-704

16. Pekmezci M, Stevers M, Phillips JJ et al (2018) Multinodular and vacuolating neuronal tumor of the cerebrum is a clonal neoplasm defined by genetic alterations that activate the MAP kinase signaling pathway. Acta Neuropathol 135:485-488

17. Pekmezci M, Villanueva-Meyer JE, Goode B et al (2018) The genetic landscape of ganglioglioma. Acta Neuropathol Commun 6:47

18. Qaddoumi I, Orisme W, Wen J et al (2016) Genetic alterations in uncommon low-grade neuroepithelial tumors: BRAF, FGFR1, and MYB mutations occur at high frequency and align with morphology. Acta Neuropathol 131:833-845

19. Ramkissoon LA, Horowitz PM, Craig JM et al (2013) Genomic analysis of diffuse pediatric low-grade gliomas identifies recurrent oncogenic truncating rearrangements in the transcription factor MYBL1. Proc Natl Acad Sci U S A 110:8188-8193

20. Rodriguez FJ, Tihan T, Lin D (2014) Clinicopathologic features of pediatric oligodendrogliomas: a series of 50 patients. Am J Surg Pathol 38:1058-1070

21. Suzuki H, Aoki K, Chiba K et al (2015) Mutational landscape and clonal architecture in grade II and III gliomas. Nat Genet 47:458-468

22. Valentijn LJ, Koster J, Zwijnenburg DA et al (2015) TERT rearrangements are frequent in neuroblastoma and identify aggressive tumors. Nat Genet 47:1411-1414

23. Zhang J, Wu G, Miller CP et al (2013) Whole-genome sequencing identifies genetic alterations in pediatric low-grade gliomas. Nat Genet 45:602-612

Ready to submit your research? Choose BMC and benefit from:

- fast, convenient online submission

- thorough peer review by experienced researchers in your field

- rapid publication on acceptance

- support for research data, including large and complex data types

- gold Open Access which fosters wider collaboration and increased citations

- maximum visibility for your research: over $100 \mathrm{M}$ website views per year

At $\mathrm{BMC}$, research is always in progress.

Learn more biomedcentral.com/submissions 